\title{
Raised CA19-9 in Autoimmune Pancreatitis
}

\author{
Sridhar Sundaram¹ Deepak Kumar Gupta ${ }^{1}$
}

\author{
${ }^{1}$ Department of Gastroenterology, Seth Gordhandas Sunderdas \\ Medical College, King Edward Memorial Hospital, Mumbai, \\ Maharashtra, India
}

\begin{abstract}
Address for correspondence Sridhar Sundaram, MD, DM, Department of Gastroenterology, Seth Gordhandas Sunderdas Medical College, King Edward Memorial Hospital, Dr. E. Borges Road, Parel, Mumbai 400012, Maharashtra, India (e-mail: drsridharsundaram@gmail.com).
\end{abstract}

\begin{abstract}
Keywords

- autoimmune pancreatitis

- CA19-9

- carcinoma of the pancreas

Autoimmune pancreatitis (AIP) is a known autoimmune disorder of the pancreas, with a clinical presentation similar to that of carcinoma of the pancreas. CA19-9 levels can be the distinguishing feature between the two disorders. In rare instances, CA19-9 may be elevated in AIP. Elevation of levels of more than $1,000 \mathrm{U} / \mathrm{mL}$ has been reported in only one case series previously. Here we report a rare instance of CA19-9 more than $1,000 \mathrm{U} / \mathrm{mL}$ in a case of AIP and its subsequent management.
\end{abstract}

\section{Introduction}

Autoimmune pancreatitis (AIP) type I is a type of chronic pancreatitis associated with raised immunoglobulin G4 (IgG4) levels in the serum, with typical histological findings and lymphoplasmacytic inflammation in the pancreas. Differential with similar clinical presentation includes carcinoma of the pancreas. Apart from imaging criteria, serum IgG4 levels, and typical histology, CA19-9 helps in distinguishing the two, with levels being raised in carcinoma of the pancreas.

\section{Case Report}

A 60-year-old male, chronic smoker, with IgG4-related nasopharyngeal mass diagnosed 8 months prior and on maintenance prednisolone $5 \mathrm{mg}$ daily presented with progressive jaundice associated with pruritus without fever since 15 days. On investigation, serum bilirubin was $13 \mathrm{mg} / \mathrm{dL}$. Ultrasonography of the abdomen showed a distended gallbladder with intrahepatic biliary radicle and common bile duct dilatation (CBD), with a suspicious hypodense mass of $3.5 \mathrm{~cm}$ in the head of the pancreas. Contrast-enhanced computed tomography (CT) scan of the abdomen showed dilated CBD with an ill-defined mass of $3.6 \mathrm{~cm}$ in the head of the pancreas, abutting the superior mesenteric vein and the portal vein, without any metastases. CA19-9 was $123.4 \mathrm{U} / \mathrm{mL}$. Endoscopic ultrasound showed a sharp and well-defined hypoechoic lesion in the head of the pancreas with altered echogenicity with encasement of portal vein confluence ( - Fig. 1). Endoscopic

DOI https://doi.org/

10.1055/s-0039-3400709

ISSN 0976-5042. ultrasound (EUS) guided fine needle biopsy (FNB) showed scanty atrophic pancreatic acini, ductal cells with dense lymphoplasmacytic infiltrate, and storiform fibrosis. There was diffuse immunostaining by IgG showing plasmacytic infiltration. There were more than 10 IgG4-positive plasma cells per high power field (-Fig. 2). Endoscopic retrograde cholangiopancreatography and plastic stent placement were performed for distal CBD stricture ( - Fig. $\mathbf{3}$ ), which was negative for malignancy on brush cytology. Serum IgG4 levels were $2.9 \mathrm{~g} / \mathrm{L}$ ( $>2$ times of the upper limit of normal). Hence, the diagnosis of type 1 AIP was established. The patient was started on prednisolone $1 \mathrm{mg} / \mathrm{kg}$ and azathioprine $2 \mathrm{mg} / \mathrm{kg}$. The steroids were then tapered to $5 \mathrm{mg}$ daily, whereas azathioprine was continued. He subsequently underwent plastic stent exchange after 3 months. Serum IgG4 levels were $1.9 \mathrm{~g} / \mathrm{L}$ on follow-up. On 6 months follow-up, liver function tests were normal, and the patient was asymptomatic. However, CA19-9 increased to 5,953 U/mL. MRI (magnetic resonance imaging) of the abdomen was unremarkable and did not show the presence of any mass in the pancreas or biliary tract or any lymphadenopathy (-Fig. 4). CA19-9 levels repeated after 6 weeks increased further to $13,780 \mathrm{U} / \mathrm{mL}$. The patient was, however, asymptomatic. There was no evidence of stent block or cholangitis. EUS was similar to previous findings. In view of suspicion of malignancy with very high CA19-9, the patient was offered surgery for the pancreas. The patient, however, was not willing for surgery and wished to continue surveillance. On follow-up, CA19-9 reduced to $1,130 \mathrm{U} / \mathrm{mL}$ after 4 weeks of the last report. Repeat contrast-enhanced

(c) 2020. Society of Gastrointestinal Endoscopy of India.

This is an open access article published by Thieme under the terms of the Creative Commons Attribution-NonDerivative-NonCommercial-License, permitting copying and reproduction so long as the original work is given appropriate credit. Contents may not be used for commercial purposes, or adapted, remixed, transformed or built upon. (https://creativecommons.org/licenses/by-nc-nd/4.0/).

Thieme Medical and Scientific Publishers Pvt. Ltd. A-12, 2nd Floor, Sector 2, Noida-201301 UP, India 
CT showed a mildly bulky head and uncinate process of the pancreas. The patient was asymptomatic with no new imaging findings. He subsequently underwent stent exchange. Repeat brush cytology was negative for malignancy. Serum IgG4 levels had reduced to $1.1 \mathrm{~g} / \mathrm{L}$. On follow-up 3 months later, the CA19-9 further reduced to $780 \mathrm{U} / \mathrm{mL}$, and the patient is persistently asymptomatic with no new findings on CT imaging. An EUS was performed, which showed a persistent similar lesion of $3 \times 2 \mathrm{~cm}$, which was sampled with EUS FNB. No malignancy was seen on histopathology. A stent exchange was performed, and the patient was continued on low-dose steroids with azathioprine. On the next follow-up after 3 months ( 1 and 1.5 years after the initial diagnosis and 1 year after the initial rise in CA19-9), the CA19-9 levels were $812 \mathrm{U} / \mathrm{mL}$, with no evidence of malignancy.

\section{Discussion}

CA19-9 is used as a marker in addition to IgG4 to differentiate AIP from pancreatic adenocarcinoma. CA19-9 less than $85 \mathrm{U} / \mathrm{mL}$ with $\mathrm{IgG} 4$ more than $280 \mathrm{mg} / \mathrm{dL}$ is highly suggestive of AIP. ${ }^{1}$ CA19-9 may be elevated in benign diseases such as choledocholithiasis, idiopathic pulmonary fibrosis,

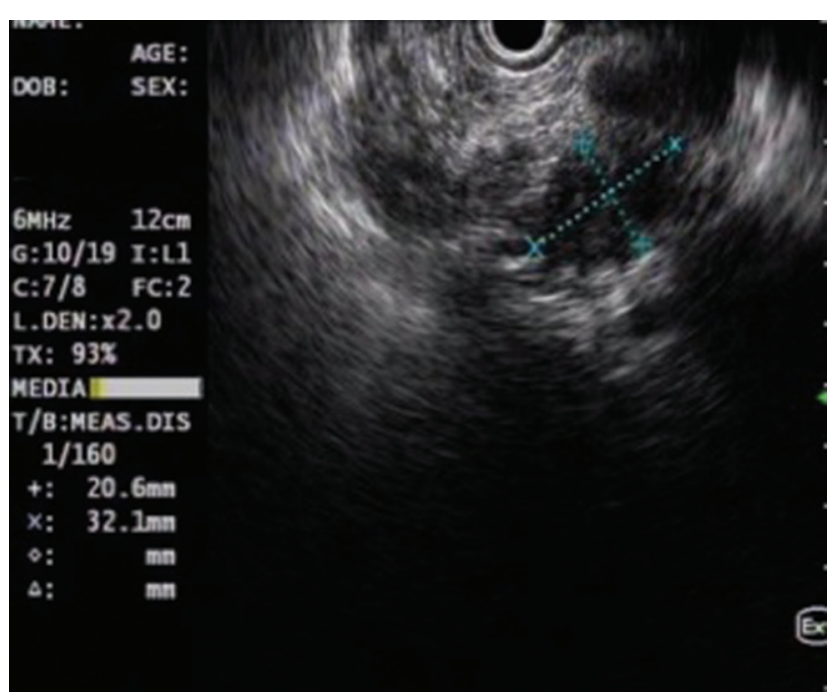

Fig. 1 Hypoechoic lesion seen on endoscopic ultrasound in the head of the pancreas.

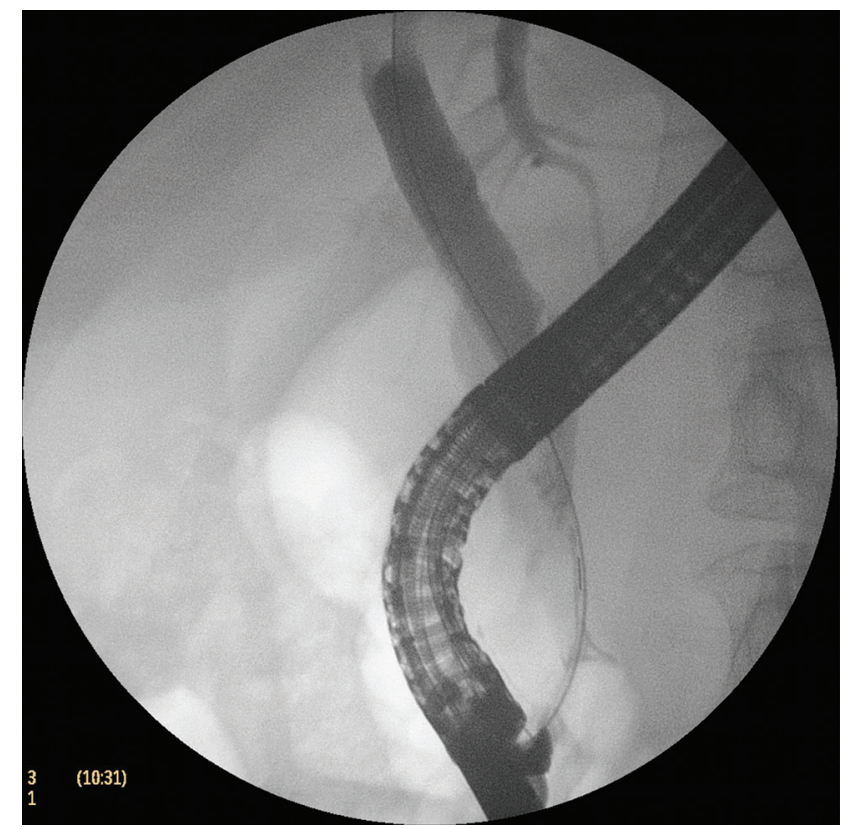

Fig. 3 Distal common bile duct dilatation stricture seen on cholangiogram during endoscopic retrograde cholangiopancreatography: brush cytology was negative for malignancy.

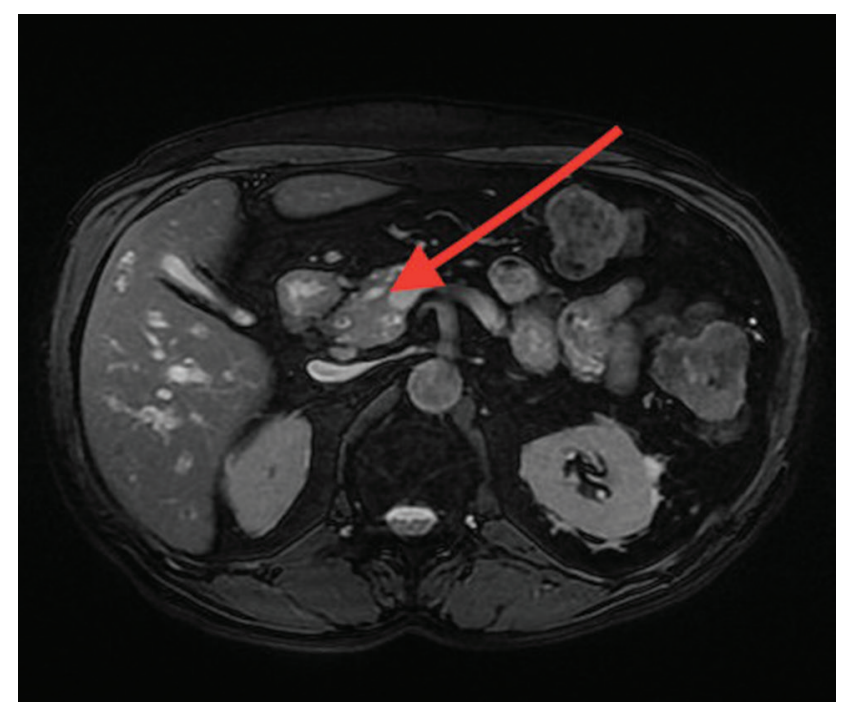

Fig. 4 MRI (magnetic resonance imaging) of the abdomen showing a mildly bulky head and uncinate process of the pancreas.

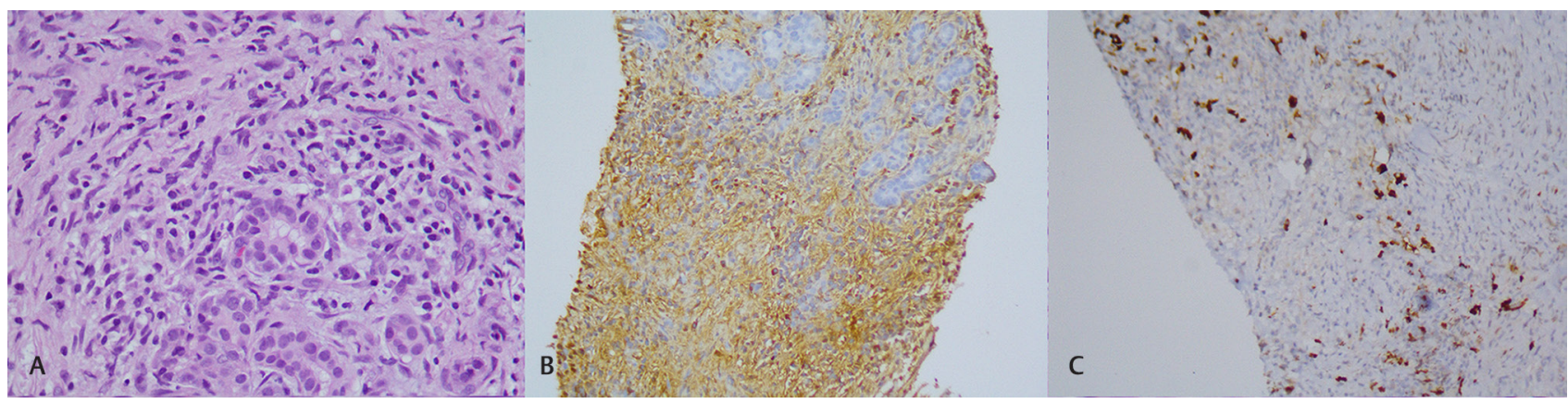

Fig. 2 (A) Hematoxylin and eosin staining of the pancreas (40x magnification) showing acini with lymphoplasmacytic infiltration and significant fibrosis. (B) Diffuse immunoglobulin ( $\mathrm{gG}$ ) immunostaining showing plasmacytic infiltration. (C) $\mathrm{lgG} 4$ immunostaining performed to confirm autoimmune pancreatitis type 1. 
interstitial lung disease, pneumonia, and hydronephrosis. ${ }^{2}$ In normal individuals, CA19-9 is produced by the biliary and pancreatic ductal epithelial cells, transported across the duct, and discharged through the duodenum. Under normal circumstances, CA19-9 is rarely released into the peripheral blood. Various mechanisms have been described in benign pancreatic and biliary diseases for the rise in CA19-9 and include the following?

- Ductal pressure in the biliary and pancreatic duct, leading to increased CA19-9 production.

- Epithelial hyperplasia due to inflammation, which leads to increased secretion of CA19-9.

- Obstruction of the biliary and pancreatic ducts with decreased clearance and reflux into the bloodstream.

- Normalization has been seen after the resolution of inflammation in conditions such as cholangitis.

In our patient, there was no evidence of active IgG4-related disease elsewhere apart from the pancreas. Hence, nonpancreatobiliary causes of raised CA19-9 were unlikely. De Marchi et al reported a series of four cases of raised CA19-9 $(>1,000 \mathrm{U} / \mathrm{mL})$ in AIP. Three patients in this series underwent surgery and had no malignancy on histopathology. Another patient responded to a steroid trial and showed a reduction in CA19-9 with treatment. ${ }^{4}$ Hence, AIP may rarely lead to increased CA19-9 in the absence of any biliary involvement.

Maintenance therapy in AIP is recommended in patients with type I AIP with diffuse enlargement of pancreas or raised $\operatorname{IgG4}$ (> 2 time the upper limit of normal) prior to treatment, delayed radiological remission, more than two other organ involvement, or proximal IgG4 sclerosing cholangitis in association with AIP. ${ }^{5}$ Our patient had a history of IgG4-related nasopharyngitis with de novo AIP developing while on low-dose maintenance corticosteroids. Hence, immunomodulators were used as maintenance in our patient after remission with prednisolone.

Surgery in AIP is recommended only for debulking or palliative bypass or in cases that do not show response to medical therapy. Surgery may help achieve clinical remission. In addition, surgery is associated with decreased chances of relapse in patients with AIP., ${ }^{5,6}$ In our patient, although surgery was planned initially in view of suspicion of malignancy, it was not performed as the patient was not willing for surgery. However, during follow-up, there was a spontaneous reduction in CA19-9 over 3 to 6 months. Another important consideration is whether AIP is a risk factor for pancreatic cancer. AIP patients who have undergone pancreatic resections show an increased propensity for pancreatic intraepithelial neoplasia. ${ }^{7}$ The incidence of pancreatic cancer ranges from 0 to $4.8 \%$ over a follow-up of 33 to 75 months in different cohorts, suggesting an increased risk in patients with AIP. ${ }^{8}$ Our patient, however, did not have any evidence of malignancy on imaging and tissue sampling.

To conclude, unless there is unequivocal evidence of malignancy in the background of AIP, raised CA19-9 may be due to background pancreatic inflammation. Surgery for pancreatic malignancy is associated with significant morbidity and mortality. Optimizing immunosuppression may avoid unnecessary surgery in this cohort with raised CA19-9 in AIP.

\section{Conflicts of Interest}

None declared.

\section{Acknowledgments}

None.

\section{References}

1 Chang MC, Liang PC, Jan S, et al. Increase diagnostic accuracy in differentiating focal type autoimmune pancreatitis from pancreatic cancer with combined serum IgG4 and CA19-9 levels. Pancreatology 2014;14(5):366-372

2 Ito S, Gejyo F. Elevation of serum CA19-9 levels in benign diseases. Intern Med 1999;38(11):840-841

3 Sheen-Chen SM, Sun CK, Liu YW, Eng HL, Ko SF, Kuo CH. Extremely elevated CA19-9 in acute cholangitis. Dig Dis Sci 2007;52(11):3140-3142

4 De Marchi G, Paiella S, Luchini C, Capelli P, Bassi C, Frulloni L. Very high serum levels of CA 19-9 in autoimmune pancreatitis: Report of four cases and brief review of literature. J Dig Dis 2016;17(10):697-702

5 Okazaki K, Chari ST, Frulloni L, et al. International consensus for the treatment of autoimmune pancreatitis. Pancreatology 2017;17(1):1-6

6 Sah RP, Chari ST, Pannala R, et al. Differences in clinical profile and relapse rate of type 1 versus type 2 autoimmune pancreatitis. Gastroenterology 2010;139(1):140-148, quiz e12-e13

7 Gupta R, Khosroshahi A, Shinagare S, et al. Does autoimmune pancreatitis increase the risk of pancreatic carcinoma?: a retrospective analysis of pancreatic resections. Pancreas 2013;42(3):506-510

8 Ikeura T, Miyoshi H, Shimatani M, Uchida K, Takaoka M, Okazaki K. Long-term outcomes of autoimmune pancreatitis. World J Gastroenterol 2016;22(34):7760-7766 